\title{
Vitalité actuelle et passée du sapin (Abies alba Mill) dans le Jura. Étude dendroécologique
}

\author{
GD Bert *, HM Becker \\ avec la collaboration technique de R Schipfer \\ INRA , laboratoire de phyto-écologie forestière, centre de recherches de Nancy, BP 35, \\ Champenoux, 54280 Seichamps, France
}

(Reçu le 20 mars 1990; accepté le 29 mai 1990)

\begin{abstract}
Résumé - A la suite des travaux sur le dépérissement du sapin (Abies alba Mill) dans les Vosges, aux sols en moyenne très acides, des recherches écologiques et dendrochronologiques ont été entreprises sur la même espèce dans un contexte phytogéographique très différent, le massif du Jura, aux sols souvent calcaires. L'article présente les premiers résultats obtenus à la suite d'observations et de prélèvements effectués en 1987. L'état sanitaire, apprécié par la notation de l'indice de transparence du houppier $/ t \%$, montre que $5,6 \%$ des sapins ont une défoliation supérieure à $25 \%$, soit 2 fois moins que dans les Vosges en 1984. Les 6 sapins dominants constituant chacune des 87 placettes ont été carottés à çeur à 1,30 m ; la mesure des largeurs de cernes sur les 522 carottes, a permis de montrer qu'en moyenne, la croissance radiale du sapin, entre 100 et 150 ans, est presque 2 fois plus forte dans le Jura que dans les Vosges. Les largeurs de cernes (en mm) ont été transformées en indices de croissance (en \%) pour les dégager de l'influence de l'âge (standardisation). La courbe moyenne de croissance radiale, entre 1828 et 1986, montre : de fortes variations interannuelles, en relation avec les conditions climatiques ; des crises, de durée et intensité variables, liées à des stress climatiques prolongés $(1922,1948$ et 1976); une tendance à long terme ascendante entre 1870 et $1930:+140 \%$ d'augmentation (dans les Vosges : $+70 \%$ ) ; cette tendance est interprétée comme une conséquence de modifications climatiques globales liées à l'augmentation du $\mathrm{CO}_{2}$ atmosphérique (effet de serre). La croissance actuelle est inversement proportionnelle au manque d'aiguilles, et ceci, dès les faibles défoliations. La croissance radiale observée entre 1940 et 1986 est proportionnelle à la largeur d'aubier, qui est ainsi un bon estimateur de la vitalité actuelle et passée des arbres. La croissance est également proportionnelle à la longueur relative de houppier, indicatrice de l'état concurrentiel moyen au long de la vie d'un arbre. La stratification des données en fonction de ces 3 critères a produit des faisceaux de courbes divergents depuis 1915-1933, soit 15-30 ans avant la forte augmentation des émissions de polluants dans l'atmosphère. Ceux-ci seraient donc, en fait, des facteurs prédisposants ou aggravants vis-à-vis des stress climatiques et/ou de mauvaises conditions sylvicoles. La suite de l'étude prévoit un renforcement de l'échantillonnage et une analyse détaillée du rôle des facteurs stationnels et climatiques sur la croissance du sapin et l'expression des phénomènes de dépérisement.
\end{abstract}

dépérlssement / dendrochronologle / climat / Ables alba / Jura

Summary - Present and past vitality of sliver fir (Ables alba Mill) in the Jura mountains. A dendroecological study. Following the studies on silver fir decline in the Vosges mountains of North-Eastern France where the soils are often very acid, ecological and dendrochronological re-

- Correspondance et tirés à part 
search has been undertaken on the same species in a different phytogeographical context: the Jura mountains, where the soils are often calcareous. This article reports the initial results derived from observations and sampling made in 1987. Health assessed by the needle loss index 1 t\% showed that $5.6 \%$ silver firs lost $25 \%$ of their needles, ie half as many as in the Vosges mountains in 1984. Eightyseven sites have been sampled throughout the natural range of silver fir in the Jura mountains, where 6 dominant trees were bored to the pith at breast height. Stand age was selected from 30 to $270 \mathrm{yr}$. The ring widths of the 522 cores were measured and crossdated. The mean radial growth, from 100$150 \mathrm{yr}$ old, was nearly twice as great in the Jura mountains as that in the Vosges mountains (fig 1). The ring widths $(\mathrm{mm})$ were then converted into growth indices (\%) with reference to the mean curve ring width vs, cambial age, to free them from the current age influence (standardization). The radial growth mean curve from 1828 to 1986 showed (fig 2): strong annual variations, in relationship with annual climatic conditions ; several crises, of varying severity and length (on the decade time scale), during which growth declined because of sustained climatic stress (1922, 1948 and 1976) (fig 3); an increasing long-term trend from 1870 to $1930:+140 \%$ for the growth index $1+70 \%$ in the Vosges mountains); this trend is interpreted to be a result of global climatic changes in relationship with the increase in atmospheric $\mathrm{CO}_{2}$ (greenhouse effect). The present growth is inversely proportional to needle loss index; this was observed even in cases of weakest needle loss. From 1940 to 1987, the radial growth was directly porportional to sapwood width. Thus, this criterion is an efficient assessment of present and past vitality of a silver fir. The radial growth is also directly proportional to the relative crown length, which is an assessment of the mean competitive status throughout the tree's life. By stratification of the sample according to these 3 previous criteria, 3 sets of divergent curves have been plotted (figs 4-6). Their divergence occurred in 1915-1933, ie 15-30 yr before the great increase of pollutant emissions into the atmosphere. Therefore, these pollutants in fact appear to be predisposing or aggravating factors towards climatic stress and/or unfavourable sylvicultural conditions. This research will be continued by examining a wider sample and studying closely the influence of ecological and climatic factors on siver fir radial growth and on the expression of forest decline.

dieback / dendrochronology / Abies alba / Jura

\section{INTRODUCTION}

Le dépérissement des forêts, plusieurs fois décrit au cours des 2 derniers siècles et d'origine inconnue, a été observé dans les Vosges à partir de 1983, particulièrement sur le sapin : Abies alba (Mill). $\mathrm{Ce}$ problème d'environnement international a suscité depuis 1984, tant en Europe qu'en Amérique du Nord, un vaste ensemble de recherches pour en déterminer les causes. En France, un des volets du programme de recherche DEFORPA (Dépérissement des forêts attribué à la pollution atmosphérique) concerne l'étude des causes "naturelles", notamment en caractérisant l'incidence des conditions écologiques sur le dépérissement. Des études dendroéco- logiques, associant étroitement étude phytoécologique et étude dendrochronologique, sont susceptibles, sous réserve d'un échantillonnage judicieux et d'un traitement approprié des données, d'évaluer l'inpact de la pollution sur la croissance radiale des arbres et de dater les phénomènes. Elles permettent aussi d'analyser la responsabilité des causes autres que la pollution atmosphérique (faible fertilité des sols, manque de réserves en eau, conditions écologiques et sylvicoles des peuplements...), soit qu'elles agissent seules, soit qu'elles renforcent l'action des polluants. Les premières études ont été engagées dans les Vosges, tant sur le sapin (Becker, 1985) que sur l'épicéa. Elles ont permis, en particulier, de montrer le rôle important 
joué par le climat dans les phénomènes étudiés, notamment lors de sécheresses provoquant un dysfonctionnement de la physiologie du sapin (Becker, 1987; Becker et Lévy, 1988). C'est dans une optique comparable que la présente étude s'est engagée, mais en choisissant une région très différente quant à la nature des roches mères, des sols et, dans une moindre mesure, quant au climat : la partie française du massif du Jura. Le sapin pectiné, essence indigène du Jura de grande importance économique, montre également des symptômes de dépérissement dans cette région. Il a donc été choisi, d'une part afin de pouvoir établir des comparaisons entres ces premiers résultats et ceux de l'étude sur la sapinière vosgienne, d'autre part parce que cette espèce est abondante sur des stations variées de la plus grande partie du massif. Cet article présente les premiers résultats relatifs à l'état sanitaire actuel des sapinières, à ses rapports avec la croissance radiale au cours des dernières décennies et à l'évolution du niveau moyen de croissance radiale depuis le début du XIX甲 siècle.

\section{AIRE D'ÉTUDE, MÉTHODES}

\section{Limites géographiques; choix des placettes}

Afin de prendre en compte toute la diversité stationnelle de la sapinière jurassienne, l'aire d'étude doit correspondre à l'aire de répartition du sapin dans le massif du Jura. La limite des forêts résineuses est proche du tracé de l'isotherme $0^{\circ} \mathrm{C}$ en janvier, qui révèle celle de l'enneigement de longue durée. La zone d'étude actuelle s'étend sur les départements du Doubs et du Jura; elle est limitée au nord et à l'est par la frontière suisse, au sud par la vallée de la Bienne près de Saint-Claude, soit environ $150 \times$ $30 \mathrm{~km}$. Chacune des 87 placettes est constituée d'un groupe de 6 sapins dominants ou codominants localisés sur une surface écologiquement homogène. Au sein des peuplements adéquats, le choix de l'emplacement précis de la placette ne tient aucunement compte de l'état sanitaire des sapins, pour que l'échantillonnage reste neutre vis-à-vis du dépérissement. L'âge du peuplement dominant est relativement homogène au sein de chaque placette, mais aussi diversifié que possible entre les placettes, pour des raisons méthodologiques propres à l'étude dendrochronologique. Ce choix est réalisé en sillonnant le massif lors de la phase de prospection sur le terrain. Les placettes sont implantées de manière à équilibrer l'échantillon vis-à-vis de l'âge des peuplements et des conditions stationnelles et orographiques.

\section{Observations phytoécologiques}

La description du site d'implantation de chaque placette est réalisée grâce à un relevé floristique et à l'aide des données stationnelles suivantes : altitude (de $550 \mathrm{~m}$ à $1300 \mathrm{~m}$ ), position topographique, profondeur et type de sol (types décrits par Bruckert et Tan, 1986; Gaiffe et Schmitt, 1980; Bruckert et Gaiffe, 1980), azimut magnétique $\left(0^{\circ}\right.$ à $\left.360^{\circ}\right)$, pente (de $0^{\circ}$ à $35^{\circ}$ ), masque (pente de la droite joignant la station étudiée au faîte du versant opposé). Pour une altitude donnée, ces 3 derniers paramètres sont intégrés en une seule grandeur : $I r=$ indice de rayonnement direct, qui traduit le climat radiatif local (lumineux et thermique) [Becker, 1984]. Les données floristiques ont été soumises à des analyses factorielles des correspondances (AFC) et des classifications ascendantes 
hiérarchiques (CAH). La répartition des espèces sur le plan factoriel $1 \times 2$ de l'AFC a permis de caractériser un gradient d'altitude sur l'axe 1 et un gradient d'hygrophilie sur l'axe 2 (Bert, 1988). Les coordonnées des relevés sur ces axes sont prises respectivement comme indice altitudinal $/ f_{1}$ et comme indice d'alimentation hydrique $I f_{2}$

\section{Observations sur les arbres}

Diverses mesures dendrométriques ont été réalisées sur les 522 arbres de l'échantillon : longueur relative de houppier Lh (mesurée au dendromètre BlumeLeiss), circonférence à $1,30 \mathrm{~m} \mathrm{C}$, concurrence en cime $\mathrm{Cm}$ (pourcentage de la couronne en contact avec les couronnes voisines). L'état sanitaire est exprimé en pourcentage d'aiguilles manquantes par rapport à la quantité que l'arbre devrait posséder s'il était parfaitement sain. Selon les auteurs, cette proportion est encore nommée «indice de transparence des houppiers" (It), «perte d'aiguilles" ou “manque d'aiguilles". La notation en pourcentage est la même que celle utilisée pour le suivi des transects DEFORPA. La part de subjectivité inhérente à l'estimation de la défoliation It a été maintenue constante par la caractérisation de tous les arbres par le même notateur.

Chaque sapin est carotté à cœur à $1,30 \mathrm{~m}$ du sol à l'aide d'une tarière de Pressler. Au laboratoire, les carottes subissent l'opération de planage : le tiers de l'épaisseur de ces cylindres de $5 \mathrm{~mm}$ de diamétre est tranché au rasoir; le plan de coupe fait apparaître nettement les limites entre cernes. La largeur d'aubier a été mesurée sur les carottes grâce à une coloration à l'acide perchlorique dilué à $40 \%$ (Kutscha et Sachs, 1962).

Les 44813 cernes des 522 carottes ont été mesurés à l'aide d'un système vidéo informatisé (précision théorique : 1/100 $\mathrm{mm}$ ). Il est ensuite essentiel de vérifier, par interdatation, tous les profils dendrochronologiques pour que chaque cerne corresponde bien à la date réelle de son élaboration. En effet, le profil peut être décalé sur l'échelle du temps par des erreurs de mesures, par des faux-cernes (rares) et surtout par des cernes manquants : pendant une ou plusieurs années, l'arbre ne produit pas de bois sur le secteur de tronc traversé par la carotte. Le principe de l'interdatation, encore nommée synchronisation, repose sur l'utilisation de quelques années ou séquences d'années caractéristiques pendant lesquelles les arbres présentent presque systématiquement une forte croissance (pic sur le profil) ou, au contraire, une croissance très faible (creux sur le profil). Le calcul de la fréquence avec laquelle chaque séquence se rencontre, au sein du lot de carottes, permet de détecter objectivement les années caractéristiques et de les hiérarchiser selon leur degré de solidité.

En moyenne, la tendance générale des dendrochronogrammes montre une diminution des largeurs de cerne au fil des ans (Fritts, 1976). Afin de pouvoir comparer des cernes d'âges différents, cette décroissance biologique normale est prise en compte par l'emploi d'une technique de standardisation. La première étape consiste à rechercher la largeur de cerne moyenne ou normale pour chaque année d'âge. Cela revient à tracer la courbe moyenne de croissance radiale en fonction de l'âge du sapin (fig 1, courbe Jura). Le point correspondant à l'âge de 20 ans, par exemple, est calculé en faisant la moyenne des centaines de largeurs de cernes élaborés par les arbres de l'échantillon quand ils avaient 20 ans. Comme chaque âge a été obtenu à des dates très variées, dans des conditions stationnelles et individuelles très différentes, la courbe 


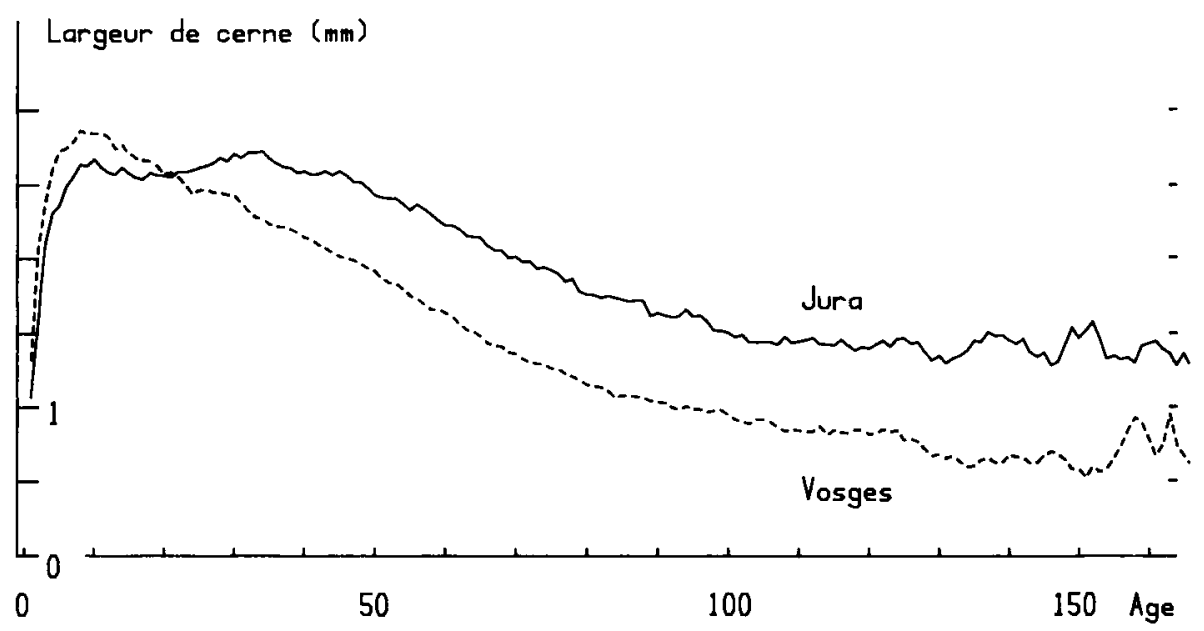

Fig 1. Accroissement moyen selon l'âge cambial de l'arbre : pour 522 sapins du Jura français, pour 1176 sapins des Vosges.

moyenne exprime essentiellement la loi biologique liant la croissance radiale au degré de vieillissement du sapin jurassien. L'ajustement mathématique de cette courbe permet de définir, pour chaque âge, un accroissement moyen théorique $A_{m}$ Connaissant l'âge de chaque cerne, l'étape de standardisation proprement dite consiste à calculer le rapport de sa largeur mesurée $L_{m}$ sur la largeur théorique à cet âge $A_{m}$ Le pourcentage obtenu est appelé indice de croissance : $I_{c}=L_{m} / A_{m} \times 100$; ces indices de croissance, dégagés de l'influence de l'âge, peuvent être utilisés pour comparer des dendrochronogrammes entre eux. L'échantillon total peut être stratifié, selon divers critères (l'intensité de la défoliation par exemple), en plusieurs sous-ensembles pour chacun desquels la courbe moyenne d'indice de croissance est calculée et comparée avec les courbes des autres sous-ensembles.

\section{RÉSULTATS}

\section{Etat sanitaire des houppiers}

Les notes de défoliation révèlent que $46,2 \%$ des sapins dominants ont un manque de feuillage inférieur ou égal à $10 \%$ (arbres considérés comme sains), 72,4\% ont un manque inférieur ou égal à $15 \%$ et seulement $5,6 \%$ d'entre eux ont un indice de défoliation supérieur à $25 \%$ (tableau I). En moyenne, l'ensemble de l'échantillon a un indice de transparence de 14,4\% (écart type $=5,1$ ). En première approche, il apparaît que la sapinière jurassienne est peu dépérissante dans son ensemble, mais comporte localement des sujets qualifiés de moyennement à fortement détériorés selon les normes préconisées par la CEE (Buffet, 1987). 
Tableau I. Effectifs selon la note de défoliation pour $\mathbf{5 2 2}$ sapins du Jura français.

\begin{tabular}{lcccccccccc}
\hline Note de défoliation (\%) & 5 & 10 & 15 & 20 & 25 & 30 & 35 & 40 & 45 & 50 \\
\% sapins (n=522) & 15,7 & 30,5 & 26,2 & 14 & 8 & 5 & 0 & 0,4 & 0 & 0,2 \\
\hline
\end{tabular}

À titre de comparaison, citons les résultats obtenus sur le massif vosgien en 1984, avec la même technique pour le choix des placettes et la notation de l'état sanitaire des sapins. L'indice de transparence moyen des 1200 sapins vosgien était de $34,3 \%$, les indices moyens par placette variaient de 15 à $53 \%$ (5 à $30 \%$ dans le Jura). Les sapins jurassiens sont globalement 2 fois moins défoliés en 1987 que ne l'étaient les sapins vosgiens en 1984. L'écart de 3 années ne peut seul expliquer cette différence : il n'a pas été signalé dans le Jura, en 1984, de peuplements aussi dépérissants qu'en certains sites vosgiens (massif du Donon, col du Bonhomme...). Le moindre dépérissement de la sapinière jurassienne est une première indication révélant des différences de comportement entre les sapins des 2 massifs.

\section{Croissance radiale}

\section{Cernes manquants; années caractéristiques}

Les carottes de sapins jurassiens comportent de nombreuses séquences de cernes très fins (minimum $=0,05 \mathrm{~mm}$ ) mais une très petite proportion de vrais cernes manquants. La différence d'intensité de dépérissement entre Vosges et Jura semble parallèle à la différence de pourcentage de cernes manquants (Elling, 1987; Wätzig et Fischer, 1987; tableau II).

Les principales années caractéristiques, mises en évidence par le calcul de leur fréquence, ont été, d'une part, des années à forte croissance : 1897, 1916, 1925, 1932, 1955, 1961, 1969, 1985, etc, d'autre part des années à faible croissance : $1870,1896,1922,1930,1932$, 1948, 1956, 1976, 1986, etc (tableau III).

\section{Accroissement annuel moyen en fonction de l'âge}

Le nombre important de données disponibles (44 813 cernes) explique que la courbe obtenue par simple calcul de moyennes soit très régulière (fig 1 , courbe Jura). Pendant les 10 premières années (âge à $1,30 \mathrm{~m}$ ), la croissance radiale des sapins jurassiens passe de $1 \mathrm{~mm} / \mathrm{an}$ à 2,6 $\mathrm{mm} / \mathrm{an}$. De 10 à 40 ans, la courbe révèle l'effet de concurrence des peuplements adultes sur la régénération naturelle. On peut penser que le mode de sylviculture moyen, assez proche de la futaie jardinée, est à l'origine de cette limitation de la croissance radiale chez les jeunes peuple-

Tableau II. Proportions comparées d'arbres dont certains cernes manquent, dans les Vosges et le Jura.

\begin{tabular}{|c|c|c|}
\hline \multirow[t]{2}{*}{$\begin{array}{c}\text { Nombre de cernes } \\
\text { manquants }\end{array}$} & \multicolumn{2}{|c|}{$\begin{array}{l}\text { Proportion des } \\
\text { arbres concernés }\end{array}$} \\
\hline & Jura & Vosges \\
\hline$\geq 1$ & $3,8 \%$ & $31 \%$ \\
\hline$\geq 5$ & $0,8 \%$ & $12 \%$ \\
\hline$\geq 10$ & $0,4 \%$ & $5 \%$ \\
\hline$\geq 15$ & $0,2 \%$ & $1 \%$ \\
\hline
\end{tabular}


Tableau III. Années et séquences d'années caractéristiques. Séquence : allure de la croissance sur 2 ou 3 années. Fréquence : celle de la séquence par rapport au nombre des carottes disponibles à cette date (nombre de carottes). Ecart $n$, écart $n+1$ : moyenne des différences de croissance radiale «année $n$ - année $n-1$ ", “année $n+1-$ année $n$ " $\left(10^{-2} \mathrm{~mm}\right)$. L'année affichée est l'année centrale dans le cas d'une séquence de 3 années, et la dernière dans le cas d'une séquence de 2 années.

\begin{tabular}{|c|c|c|c|c|c|c|}
\hline$N^{0}$ & Année & Séquence & $\begin{array}{l}\text { Fréquence } \\
\text { (\%) }\end{array}$ & $\begin{array}{r}\text { Ecart } \mathrm{n} \\
\left(10^{-2} \mathrm{~mm}\right)\end{array}$ & $\begin{array}{l}\text { Écart } \mathrm{n}+1 \\
\left(10^{-2} \mathrm{~mm}\right)\end{array}$ & $\begin{array}{l}\text { Nombre de } \\
\text { carottes }\end{array}$ \\
\hline 1 & 1925 & 1 & 89,7 & 69 & - & 467 \\
\hline 2 & 1948 & 1 & 89,5 & 59 & - & 516 \\
\hline 3 & 1986 & 1 & 88,9 & 62 & • & 522 \\
\hline 4 & 1955 & $\wedge$ & 88,9 & 60 & 106 & 521 \\
\hline 5 & 1977 & 1 & 88,5 & 55 & • & 522 \\
\hline 6 & 1970 & $i$ & 83,7 & 47 & - & 522 \\
\hline 7 & 1957 & 1 & 82,0 & 48 & - & 522 \\
\hline 8 & 1956 & V & 80,1 & $-\quad 106$ & 48 & 522 \\
\hline 9 & 1976 & V & 80,1 & $-\quad 52$ & 55 & 522 \\
\hline 10 & 1966 & 1 & 78,4 & 38 & - & 522 \\
\hline 11 & 1961 & $\wedge$ & 78,2 & 46 & 67 & 522 \\
\hline 12 & 1969 & $\Lambda$ & 78,0 & 50 & 47 & 522 \\
\hline 13 & 1932 & $\wedge$ & 77,3 & 57 & 57 & 484 \\
\hline 14 & 1940 & 1 & 76,7 & 36 & - & 507 \\
\hline 15 & 1866 & I & 76,1 & 34 & - & 88 \\
\hline 16 & 1897 & I & 75,6 & 39 & - & 266 \\
\hline 17 & 1863 & 1 & 75,3 & 27 & - & 77 \\
\hline 18 & 1922 & V & 75,1 & 43 & 46 & 450 \\
\hline 19 & 1871 & 1 & 74,2 & 32 & • & 97 \\
\hline 20 & 1917 & 1 & 74,2 & 34 & - & 426 \\
\hline 21 & 1889 & 1 & 72,9 & 37 & - & 207 \\
\hline 22 & 1982 & 1 & 71,6 & 28 & - & 522 \\
\hline 23 & 1946 & 1 & 71,6 & 28 & - & 515 \\
\hline 24 & 1868 & 1 & 70,3 & 35 & - & 91 \\
\hline 25 & 1985 & $\wedge$ & 67,8 & 36 & - & 522 \\
\hline
\end{tabular}

ments. De 40 à 130 ans, la croissance diminue progressivement jusqu'à la valeur de $1,4 \mathrm{~mm} / \mathrm{an}$ et s'y maintient. La courbe obtenue pour les Vosges (fig 1, courbe Vosges) ne révèle pas d'effet de concurrence sur les jeunes peuplements (futaies plus équiennes), mais la croissance radiale devient rapidement inférieure de 0,6 $\mathrm{mm} / \mathrm{an}$ à celle constatée dans le Jura; le cerne élaboré à l'âge de 130 ans est en moyenne environ 2 fois plus étroit que celui élaboré dans le Jura au même âge. On comprend donc la nécessité d'établir cette loi biologique pour chaque grand massif forestier si la sylviculture, les substrats ou les conditions climatiques diffèrent notablement.

Évolution de la croissance radiale de la sapinière jurassienne au cours des $\mathbf{1 5 0}$ dernières années

La courbe de la figure 2 est construite en calculant la moyenne de tous les indices de croissance disponibles pour chaque année. Du fait du grand nombre de données et des caractéristiques de l'échan- 


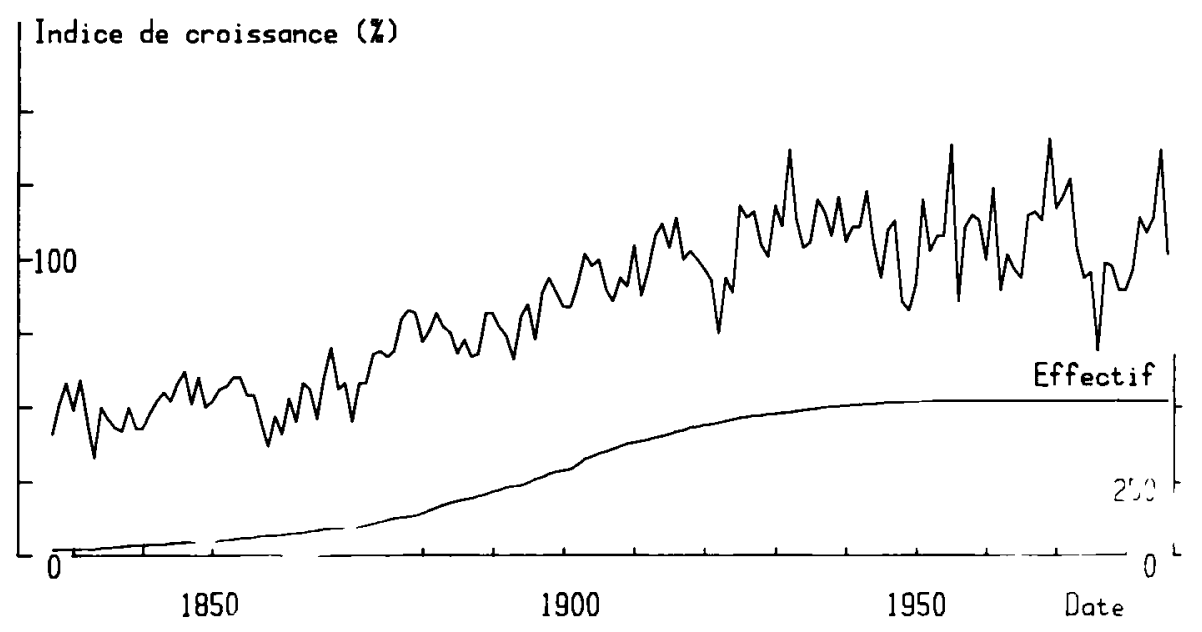

Fig 2. Évolution moyenne des indices de croissance radiale $l c(\%)$ du sapin dans le Jura français pendant la période 1828-1986 et effectif de l'échantillon selon la date.

tillonnage, cette courbe est statistiquement très fiable, tant dans ses fluctuations instantanées que dans son allure générale. Ce profil de référence n'est valable, en toute rigueur, que pour l'espèce Abies alba, dans le massif jurassien. En effet, chaque essence a des exigences qui lui sont propres et il serait imprudent d'extrapoler ces résultats à d'autres espèces. La spécificité géographique est également importante : les accidents climatiques ne surviennent pas toujours les mêmes années dans les diverses régions.

\section{Les crises}

Bien que restant en moyenne proche de 110 à $120 \%$ de l'accroissement moyen, la croissance a connu plusieurs périodes de dépression ou crises au cours du XXe siècle. Pendant celles-ci, les sapins ont élaboré des cernes très étroits. Des crises de faible durée ou intensité s'observent en 1911, 1933, 1956, 1962, 1965. D'autres crises, plus importantes en durée et intensité, se situent pendant les périodes
1916-1925 (très marquée en 1922), 19431951 (accusée en 1948 et 1949) et 19731982 (centrée sur 1976). Pendant cette dernière, la croissance radiale a commencé à se réduire dès 1973 , pour n'être plus que de $70 \%$ de l'accroissement moyen en 1976. Les années 1977 et 1978 montrent un début de rétablissement auquel succède une rechute en 1979-1980, plus marquée dans le Jura que dans les Vosges. La croissance radiale revient au niveau moyen du XXe siècle en 1981 et surtout 1982. L'année 1983 semble révéler une divergence entre les Vosges, où la croissance radiale s'est améliorée (jusqu'à $125 \%$ ), et le Jura, où les sapins gardaient une croissance proche de $110-115 \%$ entre 1982 et 1984.

\section{Évolution globale}

Au-delà des variations interannuelles, la période 1830 à 1986 met en évidence les grandes tendances de l'évolution de l'indice de croissance. Notons le fait marquant qu'est l'augmentation considérable 
de la croissance radiale entre 1870 et 1930. L'indice de croissance augmente progressivement de 50 (avant 1860) jusqu'à $110-120$ en 1930 , soit près de $140 \%$ de plus. Après le maximum des années trente, le niveau moyen fluctue légèrement plus bas : 100-110. Cette baisse, peu marquée dans le Jura, est plus importante sur le massif vosgien (Becker, 1987). Par contre, l'augmentation au cours des années 1870 à 1930 fut de moindre amplitude dans les Vosges (+ $70 \%$ ). La sapinière jurassienne considérée dans son ensemble ne subit donc pas de phénomène de baisse générale de vitalité, bien au contraire. La crise de ces dernières années, resituée dans un contexte plus général, se révèle peu commune mais non exceptionnelle; de plus, du point de vue de la croissance radiale, elle paraît achevée. Les sapins retrouvent à partir de 1982 un potentiel d'accroissement en diamètre proche de la moyenne du $\mathrm{XX}^{\ominus}$ siècle. Ces grands traits de l'évolution de la sapinière jurassienne sont similaires à ceux de la sapinière vosgienne, en l'état actuel des recherches. De semblables tendances à long terme sont également signalées en
Allemagne (Kontic et al, 1990; Kenk, 1987), en Finlande (Hari et al, 1984), aux États-Unis (Lamarche et al, 1984; Cooper, 1986; Hornbeck, 1987), au Canada (Jozsa et Powel, 1987; d'Arrigo et al, 1987).

\section{Climat jurassien et accroissement radial des sapins}

Parmi les stations météorologiques proches du massif, la station de Lyon-Bron dispose de relevés de précipitations et températures depuis 1921. La comparaison de ces relevés avec ceux de Besançon, plus jurassiens, montre que le sens des variations interannuelles est le plus souvent identique pour les deux stations; elles sont donc soumises au même macroclimat (fig 3). Les courbes de pluviométrie et de température annuelles ne varient pas parfaitement en phase avec la courbe de croissance de référence, probablement en raison d'arrières-effets du climat d'une année sur la croissance des arbres pendant les années suivantes (Becker, 1989). Toutefois, les effets d'années ou séries d'années particulières sont nettement mis en évidence. Une série d'années sèches

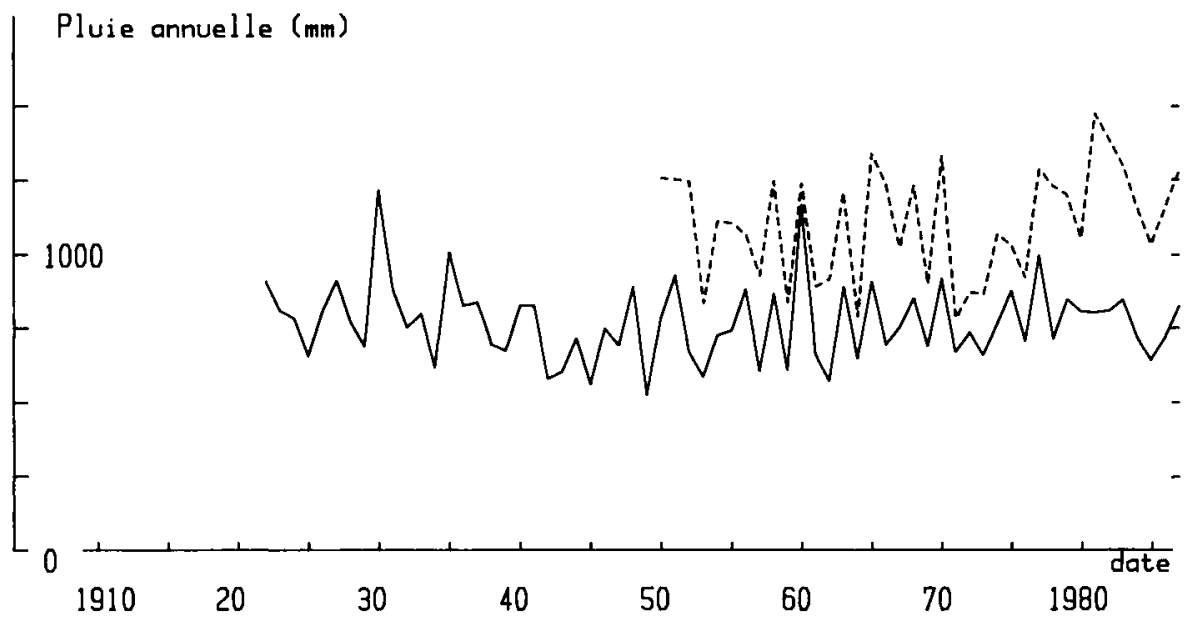

Fig 3. Précipitations annuelles à Besançon (pointillé) et à Lyon (trait plein). 
(1971-1976) a déclenché à partir de 1973 une importante diminution de la croissance radiale; on a pu en observer les conséquences par le déclenchement du phénomène appelé “dépérissement». Les années suivantes, plus humides, ont permis de meilleurs accroissements (fig 2). $\mathrm{Ce}$ type d'observation permet de comprendre les crises plus anciennes : 19621965 (années 1961, 1962 et 1964 sèches), 1956 (gel tardif), 1948-1949 (série d'années sèches entre 1942 et 1949), 1916-1925 (1921 : année la plus sèche de toute la série disponible).

Cependant cette vue d'ensemble peut occulter certains phénomènes localisés (du moins pour le moment), notamment ceux liés aux situations actuellement les plus dépérissantes. C'est pourquoi, il est prévu de traiter ultérieurement plus en détail l'incidence des facteurs écologiques sur la croissance radiale et sur l'état sanitaire et/ou le dépérissement.

\section{Relations entre la croissance radiale et d'autres caractéristiques biométriques}

Le principe de cette recherche repose sur la stratification de l'échantillon global. Pour caractériser le rôle des variables biométriques mesurées, chacune d'elle est divisée en 4-7 classes, pour lesquelles la courbe moyenne d'indice de croissance est calculée.

Parmi les paramètres mesurés sur chacun des arbres, l'indice de transparence du houppier, la largeur d'aubier, la longueur relative de houppier et la concurrence en cime s'avèrent fortement en relation avec la croissance radiale.

\section{Indice de transparence du houppier (It)}

Les 5 courbes moyennes correspondant aux 5 strates d'indices /t s'organisent selon un faisceau divergent à partir de 19181925 (fig 4). La courbe des arbres sains

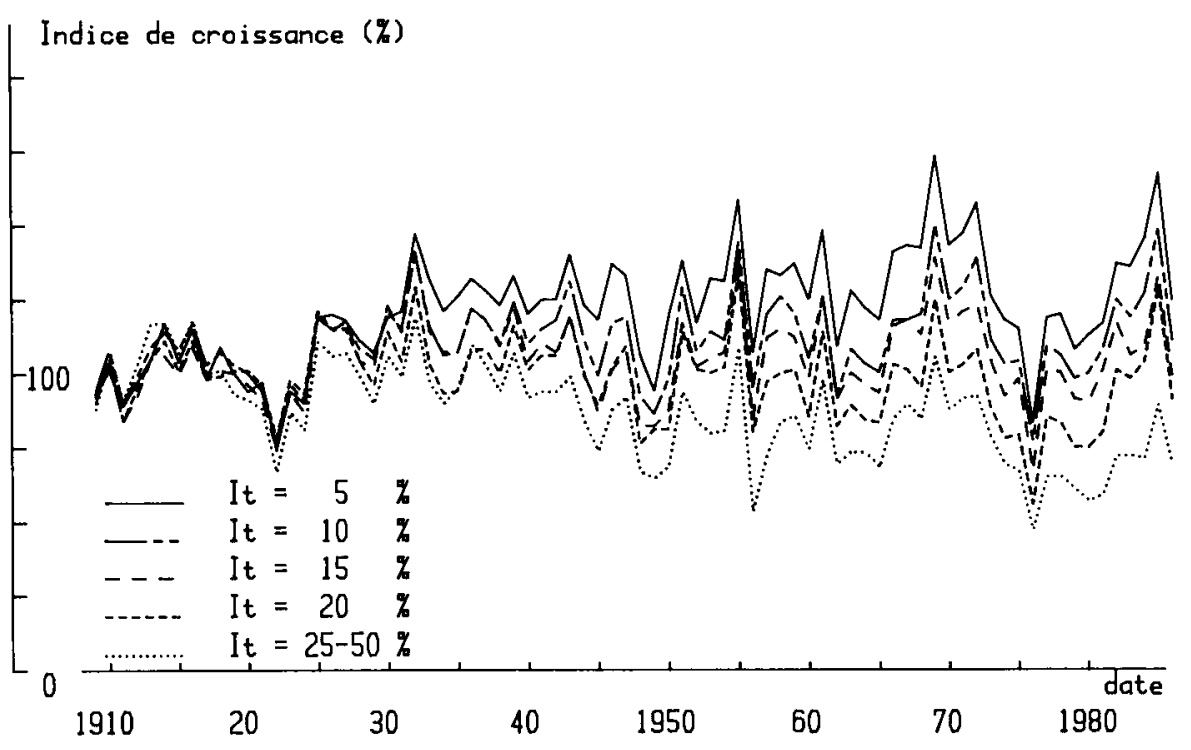

Fig 4. Évolution moyenne des indices de croissance radiale selon le manque d'aiguilles It (\%). 
( $I t=0-9 \%$ ) se situe à $20-30 \%$ au-dessus de la courbe de référence $(240 \%$ d'augmentation depuis le siècle dernier), celle des arbres légèrement plus défoliés ( $I t=$ $10-14 \%$ ) lui est inférieure de $15 \%$. Ce décalage laisse penser qu'une faible défoliation provoque déjà une perte d'accroissement en diamètre tout à fait sensible. Or, le phénomène de réduction de la croissance radiale n'avait pu être constaté sur les sapins vosgiens que pour des défoliations de l'ordre de 35-40\% (Becker, 1987). De même, Kenk (1989), en Allemagne, constate que seuls des dommages importants ont une répercussion significative sur l'accroissement. Pour le Jura, on constate que les notes de défoliation sont très discriminantes; à chaque classe de $5 \%$, correspond une perte de croissance tout à fait nette. La mesure de l'écart entre la courbe des arbres sains ( $I t=5 \%$ ) et les autres courbes fait apparaître que, dans les limites de défoliation observées (5 à $30 \%$ ), la perte de croissance est directement proportionnelle à la défoliation. Ceci rejoint les conclusions de Bräker (1987), en Suisse, qui a constaté d'une manière nette et significative du point de vue statistique que la moyenne suisse de l'accroissement des résineux étudiés est d'autant plus basse que le taux de défoliation est plus grand.

La divergence des courbes commence dans les années 1920, alors qu'elle est nettement plus tardive dans les Vosges (dans les années 1950; Becker, 1987). Elle confirme qu'il n'y a pas coïncidence avec l'augmentation brutale de la pollution atmosphérique des années 1950 (Kenk, 1989). Quelques éléments d'explication sont fournis par la comparaison de la croissance radiale des sapins de même classe de défoliation, mais situés en conditions d'alimentation hydrique variables (exprimées par l'indice $\left(f_{2}\right)$. Les arbres peu défoliés $(I t=0$ à $10 \%)$ en station humide ont une croissance forte, toujours supérieure à la moyenne; en station moins bien alimentée en eau, ils ont une croissance radiale faible au début (fin du siècle dernier) mais en augmentation constante depuis un siècle. Ce résultat suggère l'hypothèse d'une modification des conditions climatiques, à savoir une augmentation des précipitations qui atténuerait le handicap des stations les plus sèches sur le plan édaphique. La croissance des sapins les plus défoliés ( $I t>20 \%$ ) est en nette diminution depuis les années 1920-1930, moins prononcée en station plus humide, où la meilleure réserve en eau du sol limite les dégâts dus aux sécheresses. Ces observations font ressortir une fois encore l'importance fondamentale du facteur «alimentation en eau» dans l'expression de la croissance radiale.

\section{Largeur d'aubier}

La masse foliaire d'un arbre, en équilibre avec son milieu, est fortement corrélée avec la section conductrice efficace du point de vue du flux transpiratoire : le bois d'aubier (Snell et Brown, 1978). Le critère de largeur d'aubier est ainsi a priori un estimateur objectif de sa vitalité. De fait, la stratification en fonction de la largeur d'aubier fournit également un faisceau de courbes nettement divergentes (fig 5), en relation avec une baisse continue de la vitalité des sapins, depuis ceux de plus grande vigueur (aubier de $114 \mathrm{~mm}$ en moyenne) jusqu'aux plus faibles (aubier de $41 \mathrm{~mm}$ en moyenne). L'individualisation de ces divers comportements est là encore très ancienne, et se situe entre 1914 et 1933, période semblable à celle observée pour la défoliation. Le même phénomène se constate dans chaque strate de défoliation, ce qui indique que la largeur d'aubier apporte une information supplémentaire.

La perte de croissance radiale, en 1986, par rapport à la courbe des plus grandes 


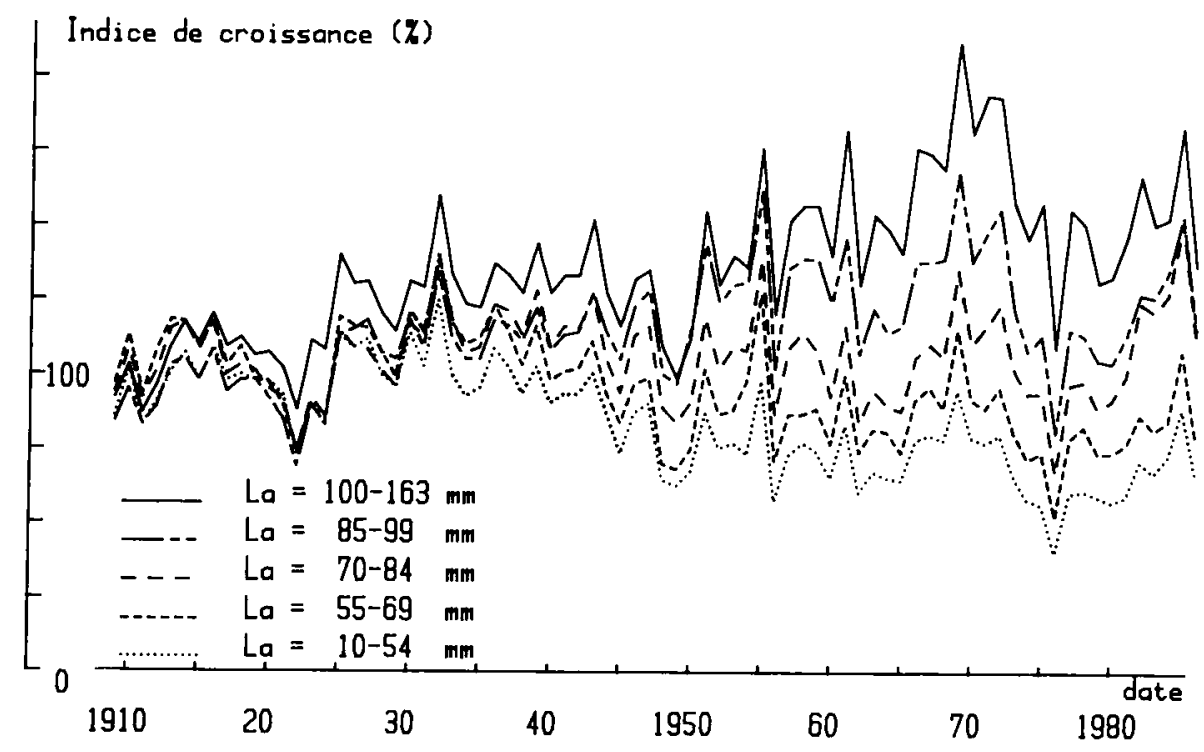

Fig 5. Évolution moyenne des indices de croissance radiale selon la largeur d'aubier La (mm).

largeurs d'aubier a été mesurée. II apparaît ainsi que la croissance radiale est directement proportionnelle à la largeur d'aubier. La relation se maintient en effectuant ces mesures pour des années de plus en plus anciennes : 1980, 1970... 1940. Elle n'est plus vérifiée pour des dates antérieures à 1940. Le critère de largeur d'aubier fournit ainsi une indication de la vitalité de l'arbre pendant une période plus longue que le critère de défoliation, lequel est susceptible de se modifier plus rapidement d'une année à l'autre.

\section{Longueur relative de houppier}

Ce critère est également en relation avec la masse foliaire actuelle, qui est le résultat de l'état concurrentiel dans lequel l'arbre a poussé depuis plusieurs décennies. La longueur relative de houppier permet donc d'estimer l'intensité de la concur- rence ancienne subie par chaque arbre. Une famille de courbes s'individualise à partie des crises 1932-1933 et 1948-1949 (fig 6). la croissance radiale des arbres à houppier long est supérieure, depuis en moyenne 60 ans, à celle des arbres à houppier court. Ce résultat est en fait en grande partie une expression de la corrélation, chez un arbre en équilibre avec son milieu, entre la masse foliaire et la largeur du bois d'aubier (Granier, 1981). Les arbres possédant un feuillage important, conséquence d'une faible concurrence interindividuelle, ont une forte capacité photosynthétique; cette vigueur leur confère, malgré les crises, une croissance radiale supérieure à celle des arbres moins feuillus. En effet, à un même taux de défoliation ne correspond pas une même quantité d'aiguilles, en valeur absolue, sur un sapin à houppier court et sur un sapin à houppier long. Ces différences morphologiques induisent des différences 


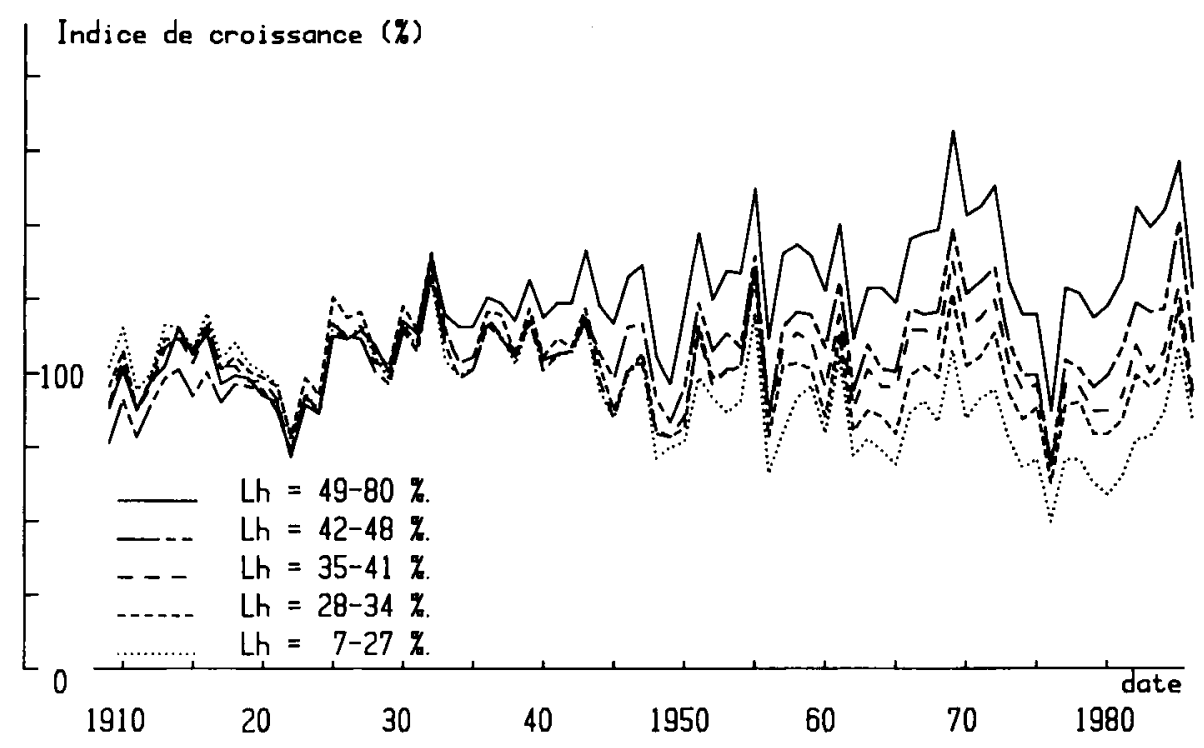

Fig 6. Évolution moyenne des indices de croissance radiale selon la longueur relative de houppier Lh (\%).

de croissance radiale et de résistance aux stress. Pour une même défoliation (inférieure à $20 \%$ ) la croissance radiale est d'autant meilleure que le houppier est long; si la défoliation est plus importante (supérieure à $20 \%$ ), la croissance radiale reste faible quelle que soit la longueur de houppier. Ainsi, bien que l'indice de transparence du houppier et la longueur relative de houppier permettent tous 2 d'obtenir des faisceaux de courbes de croissance radiale nettement divergents et qu'ils soient corrélés $(r=-0,423$; significatif au seuil de $1 \%$, mais nettement différent de 1 ), ces 2 paramètres ne sont que partiellement redondants.

\section{Concurrence en cime}

Pour un arbre donné, le pourcentage de contact entre sa couronne et celles de ses voisins traduit l'état concurrentiel dans lequel il se développe depuis quelques années (concurrence actuelle). Les sapins en contact avec leurs voisins sur plus de $80 \%$ de la circonférence de leur couronne montrent une faible croissance radiale depuis le début des années cinquante. Une forte concurrence en cime réduit, à terme, la longueur relative du houppier, avec les conséquences décrites dans le paragraphe précédent. Cette action est réversible : les sapins actuellement en contact sur moins de $20 \%$ de leur circonférence montrent une faible croissance entre 1870 et 1950, puis celle-ci revient au niveau moyen. L'observation des profils individuels de ces arbres révèle l'existence d'éclaircies entre 1925 et 1945. Ces résultats rappellent le rôle important des opérations sylvicoles vis-à-vis de la croissance radiale : la fermeture du couvert provoque un important élagage naturel, ce qui est 
bénéfique; mais au-delà d'un certain seuil, la masse de houppier devient trop faible, ce qui sensibilise les arbres aux stress climatiques.

\section{DISCUSSION ET PERSPECTIVES}

\section{Relation entre la croissance et la masse foliaire (longueur de houppier, défoliation)}

Cette relation, mise en évidence ici, a également été montrée lors d'études suisses et allemandes. Mandallaz et al (1986) ont déterminé que l'influence du houppier, lorsqu'elle existe, est favorable. Les sapins de Sainte-Croix (Suisse) à houppier long ont une plus grande probabilité d'être sains que ceux à houppier court. Le suivi basé sur 2 inventaires (1970-1984) par le Bernese Oberland Forest Service a permis d'établir une relation statitique entre la longueur relative 'de houppier, l'accroissement en diamètre et la perte d'aiguilles (Murri et Schlaepfer, 1987). Les arbres ayant de longues couronnes ont des croissances en hauteur et en diamètre supérieures et surmontent mieux les périodes de sécheresse. En moyenne, ils perdent moins d'aiguilles et maintiennent également une production plus élevée pendant une période plus longue. La longueur de la couronne se révèle ainsi être une bonne caractéristique de la vitalité d'un arbre (Spiecker, 1986). Toute une série de résultats permettent de conclure à une composante sylvicole du dépérissement, sur laquelle le forestier peut avoir une influence: longueur des couronnes, mélange des essences, structure du peuplement (Kenk, 1989; Becker et Lévy, 1988; Landmann, 1988).

\section{L'augmentation à long terme de la croissance radiale de la sapinière}

Elle est conditionnée par des facteurs en évolution qualitative ou quantitative à l'échelle du siècle. Le calcul de la fonction de réponse de la croissance des sapins vosgiens a montré l'influence prédominante des facteurs climatiques : températures et précipitations (Becker, 1989). L'augmentation de productivité des sapins, simultanée dans le Jura et dans les Vosges, s'est produite pendant une période de réchauffement de l'hémisphère Nord, de 0,4 à $0,7^{\circ} \mathrm{C}$, entre 1885 et 1940 (Jones et al, 1986). Depuis, température et croissance radiale se maintiennent à des niveaux proches du maximum historique, en accusant de fortes variations interannuelles. Ainsi, cette augmentation du niveau moyen de croissance radiale exprimerait les modifications climatiques à long terme. Les données de température du début des années 1980 laissent deviner une nouvelle accélération du réchauffement de l'atmosphère, qui serait une conséquence de l'effet de serre dû au gaz carbonique (Bach, 1985; von Grassl, 1987); l'avenir dira si la croissance radiale des sapins répondra encore par une augmentation à plus ou moins long terme... On pourrait aussi craindre qu'une dérive lente et générale des pratiques sylvicoles dans l'ensemble de la région, durant les 100 dernières années, n'ait modifié les densités moyennes des peuplements et, ainsi, biaisé l'évolution de la courbe moyenne d'indice de croissance. Hormis le fait qu'une telle dérive n'ait pas été signalée dans les sapinières jurassiennes, la fidélité de la reconstruction du même type de tendance dans les Vosges par les données climatiques, sans être une preuve irréfutable, conduit à penser que le climat est 
probablement aussi l'élément déterminant dans le Jura.

De premiers traitements faisant intervenir les données écologiques stationnelles ont montré, grâce à l'utilisation de l'indice de climat radiatif $I r$, que l'amélioration de la croissance radiale des sapins sur les stations froides a été supérieure à celle mesurée sur les stations à bioclimat plus chaud; ces dernières ont été plus sensibles aux sécheresses, surtout celles des années soixante-dix. Ces résultats sont cohérents avec l'hypothèse d'un léger réchauffement du climat jurassien, plus favorable pour les arbres des stations où les basses températures sont le facteur limitant. Le phénomène ne semble pas limité à ce cas précis : en Aliemagne, Kenk (1989) a observé que l'épicéa présente actuellement un niveau de production supérieur à ce qu'il devrait être, et ce, d'autant plus qu'on se situe dans des classes de productivité faibles.

L'augmentation de température, si minime soit-elle en moyenne, peut également avoir eu pour effet de grossir les précipitations (évaporation océanique plus importante?), ce qui favoriserait par contrecoup la végétation forestière. Sur les stations les plus sèches de l'échantillon jurassien, la croissance radiale des sapins non défoliés s'est davantage améliorée que l'ensemble de la sapinière; les faibles réserves en eau de ce type de station ont pu être complétées par des précipitations plus abondantes. Cependant, l'augmentation globale des précipitations, ainsi indirectement révélée, ne doit pas faire perdre de vue la fréquence des séries d'années sèches au cours du XXe siècle : 19441950, 1962-1965, 1972-1977. Leurs effets, contraires à l'amélioration générale de la productivité, se constatent particulièrement sur les stations à bioclimat chaud. Ces premières conclusions sont préliminaires à un projet de confrontation analyti- que plus approfondie entre la croissance radial et les facteurs climatiques par le calcul de fonctions de réponse.

Ainsi, dans le Jura comme dans les Vosges, les chocs climatiques dus aux sécheresses des années 1917-1924 et 1932-1933 ont affecté les arbres de façon plus ou moins intense selon leur niveau de vigueur intrinsèque et la densité du peuplement (Lévy et Becker, 1987). Les sapins ont exprimé ces stress climatiques sous la forme de défoliations et de réductions de croissance. Les arbres à houppier équilibré ont pu retrouver, après quelques années, un bon niveau de croissance radiale tandis que les plus concurrencés, les plus défoliés, ou ceux localisés sur les stations les plus défavorables, perdaient de plus en plus leur potentiel de croissance.

\section{Effets de la pollution atmosphérique générale}

D'éventuels effets de la pollution atmosphérique, par exemple pluies acides, n'ont pu être détectés au cours de cette étude. D'une part, l'individualisation de groupes de sapins de vigueur différente s'est produite au cours des années 1915-1933, soit 15-20 ans avant l'augmentation considérable des émissions de produits polluants dans l'atmosphère. D'autre part, certains résultats, tels que l'influence de facteurs stationnels (exposition, réserves en eau du sol), ou de la sylviculture sur l'intensité des défoliations, ne confirment pas l'hypothèse d'une action prédominante des pluies acides. L'acidification de la couverture pédologique, susceptible de se produire dans le cas d'apports atmosphériques de polluants (Bonneau et al, 1986; Falkengren-Grerup, 1987), ou une éventuelle déficience de la nutrition minérale des arbres ne se sont pas avérées corrélées avec le dépérissement à l'étage mon- 
tagnard du Jura (Bruckert et Tan, 1986). L'éventuelle action de dépôts polluants s'exercerait plutôt directement sur les feuilles des arbres et aggraverait leur état ou les sensibiliserait aux effets d'autres facteurs, tels une médiocre fertilité naturelle des sols ou des stress climatiques et pédoclimatiques, plus particulièrement hydriques (Bonneau, 1988).

\section{Pourquoi certains peuplements sont-ils aujourd'hui encore dépérissants ?}

Bien que la sapinière jurassienne, considérée dans son ensemble, montre un net rétablissement du point de vue de la croissance radiale, après une période de crise d'une quizaine d'années, nos résultats actuels indiquent que certains peuplements sont aujourd'hui encore dépérissants. Sont-ils en difficulté du fait de mauvaises conditions stationnelles ou sylvicoles? Sont-ils les signes, pour l'instant localisés, d'un réajustement de l'écosystème? Révèlent-ils une plus grande sensibilité de certains arbres ou de certaines stations aux stress climatiques ? Subissent-ils plus particulièrement les effets de pluies acides, directement ou par l'intermédiaire du sol? Ces interrogations montrent que les recherches méritent d'être approfondies pour améliorer la connaissance de l'autécologie du sapin, du fonctionnement des écosystèmes forestiers et de l'impact des divers polluants sur la santé et la productivité de la forêt. La suite de cette étude prévoit une extension du domaine d'étude à l'intégralité de l'aire de répartition du sapin dans le massif jurassien, français et suisse $(250 \times 50 \mathrm{~km})$; l'échantillonnage sera complété en augmentant le nombre de placettes jusqu'à concurrence d'environ 200, notamment au sein de sapinières sèches du versant suisse et du sud du Jura. Les nouvelles données dendroécologiques de- vraient permettre, entre autres objectifs, de comparer les modèles climatiques vosgien (Becker, 1989) et jurassien et d'affiner le rôle des conditions écologiques stationnelles dans l'expression du potentiel de croissance radiale.

\section{RÉFÉRENCES}

d'Arrigo R, Jacoby GC, Fung IY (1987) Boreal forests and atmosphere-biosphere exchange of carbon dioxide. Nature 239, 321-323

Bach W (1985) Der anthropogen gestörte Kohlenstoffkreislauf: Methoden zur Abschätzung der $\mathrm{CO}_{2}$-Entwiklung in der Vergangenheit und in der Zukunft. Düsseldorfer Geobot Kol$\log 2,3-23$

Becker M (1984) Indices de climat lumineux selon la pente et l'exposition pour les latitudes de 40 à $50^{\circ}$. Bull Ecol 15, 239-252

Becker M (1985) Le dépérissement du sapin dans les Vosges. Quelques facteurs liés à la détérioration des cimes. Rev For Fr 37, 281 287

Becker M (1987) Bilan de santé actuel et rétrospectif du sapin (Abies alba Mill) dans les Vosges. Étude écologique et dendrochronologique. Ann Sci For 44, 379-402

Becker M (1989) The role of climate on present and past vitality of silver fir forests in the Vosges mountains of Northeastern France. Can J For Res 19, 1110-1117

Becker M, Lévy G (1988) A propos du dépérissement des forêts : climat, sylviculture et vitalité de la sapinière vosgienne. Rev For Fr 40, 5, 345-358

Bert GD (1988) Étude dendroécologique du dépérissement du sapin (Abies alba Mill) dans le Jura. DEA Biol For, Univ Nancy 1,63 p + annexes

Bonneau M (1988) Étude de la nutrition minérale du sapin et d'épicéas jaunissants du Jura central et méridional. In: Journées de travail. Programme DEFORPA. Dépérissement des forêts et pollution atmosphérique. Vol 3, 6.4 bis 1-16

Bonneau $M$, Dambrine $E$, Nys $C$, Ranger $J$ (1986) L'acidification des sols. Bull Ecol 18, $127-136$ 
Bräker OU (1987) Rapport SANASILVA sur les dégâts aux forêts 1987. Off Féd For Protec Paysage, Berne. Inst Féd Rech For Birmensdorf

Bruckert S, Gaiffe M (1980) Pédogenèse en pays calcaire glaciaire ou karstique. Analyse des facteurs de formation et de distribution des sols en pays calcaire glaciaire ou karstique. Ann Sci Univ Besançon, fasc 1, $4^{\theta}$ sér Biol Vég

Bruckert S, Tan BS (1986) Étude du dépérissement des forêts de l'étage montagnard du Jura en relation avec les sols calciques ou acides du karst, l'importance de leurs éléments nutritifs et l'état nutritionnel des peuplements. In: Programme DEFORPA. État des recherches à la fin de lannée 1986. Vol 1, 133-147

Buffet $M$ (1987) Le réseau de surveillance sanitaire des forêts. Description. Problématique. In: Les recherches en France sur le dépérissement des forêts. Prog DEFORPA. $1^{\text {er rap- }}$ port, ENGREF Nancy, 11-16

Cooper CF (1986) Carbon dioxide enhancement of tree growth at high elevations. Science 231,259

Elling (von) W (1987) Eine Methode zur Erfassung von Verlauf und Grad der Schädingung von Nadelbaumbeständen. Eur J For Pathol $17,426-440$

Falkengren-Grerup $U$ (1987) Long-term changes in $\mathrm{pH}$ of forests soils in southern Sweden. Environ Pollut Ser A 43, 79-90

Fritts HC (1976) Tree-ring and climate. Acad Press, $567 p$

Gaiffe $M$, Schmitt $A(1980)$ Sols et végétation de l'étage montagnard dans les forêts du Jura central. Extraits Sci Sol, 4, 265-296

Granier A (1981) Étude des relations entre la section du bois d'aubier et la masse foliaire chez le douglas (Pseudotsuga menziesi Mirb Franco). Ann Sci For 38, 503-512

von GrassI H (1987) Klimaänderung durch erhöhte Spurenstoffgehalte in der Atmosphäre. Forstwiss Centralbl Ham 106, 236-248

Hari $P$, Arovaara $H$, Raunemaa $T$, Hautojärvi $A$ (1984) Forest growth and the effects of energy production: a method for detecting trends in the growth potential of trees. Can J For Res 14, 437-440
Hornbeck JW (1987) Growth patterns of red oak and red and sugar maple relative to atmospheric deposition. Proc 16th Central Hardwood Forest Conference (24-26 Feb 1987) Knoxville, TN, Univ Tennessee, 277-282

Jones PD, Raper SCB, Bradley RS, Diaz HF, Kelly PM, Wigley TML (1986) Northern hemisphere surface air temperature variations: 1851-1984. J Climate Appl Meteorol 25, 161179

Josza LA, Powel JM (1987) Some climatic aspects of biomass productivity of white spruce stem wood. Can J For Res 17, 1075-1079

Kenk G (1987) Der Vergleich von Wachstumsdaten aufeinander-folgender Waldbestandsgenerationen. KFK PEF 12, 87-99

Kenk G (1989) Zuwachtuntersuchungen im Zusammenhang mit den gegenwärtigen Waldschäden in Baden-Württemberg. Proc 14th Meet Spec Air Pollution Effects on For Ecosyst, Interlaken, Suisse, 2-8 Oct 1988. Birmensdorf, 263-269

Kontic R, Braeker OU, Nizon V, Mueller R (1990) Jahrringanalytische Untersuchugen im Sihlwald. Schweiz $Z$ Forstwes (sous presse)

Kutsha NP, Sachs IB (1962) Color tests for differentiating heartwood and sapwood in certain softwood tree species. US For Prod Lab Madison, Wis, FPL, no 2246, $13 p$

Lamarche VC, Graybill DA, Fritts HC, Rose MR (1984) Increasing atmospheric carbon dioxide: tree ring evidence for growth enhancement in natural vegetation. Science 225 , 1019-1021

Landmann G (1988) Les recherches sur le dépérissement en France : structure et principaux résultats du programme DEFORPA. Air Pollution and Ecosystems. Proc Int Symp 18-22 May 1987, Grenoble, France. Kluwer Acad Publ, Norwell, MA 261-281

Lévy $G$, Becker $M$ (1987) Le dépérissement des sapins dans les Vosges. Rôle primordial de déficits d'alimentation en eau. Ann Sci For $1987,44,379-402$

Mandallaz D, Schlaepfer R, Arnould J (1986) Dépérissement des forêts : essai d'analyse des dépendances. An Sci For 43, 441-458 
von Murri M, Schlaepfer R (1987) Zusammenhänge von Kroneneigenschaften und Durchmesser-bzw. Grundflächenzuwachs von Fichte auf zwei Gebirgsstandorten. Forstwiss Centrabl Ham 106, 328-340

Snell KJA, Brown JK (1978) Comparison of tree biomass indicators $\mathrm{DBH}$ and sapwood area. For Sci 24, 455-457
Spiecker H (1986) Das Wachstum der Tannen und Fichten auf Plenterwald - Versuchsflächen des Schwarzwaldes in der Zeit von 1950 bis 1984. Allg Forst Jagdtztg 157, 152164

Wätzig H, Fischer B (1987) Untersuchungen über den Jahrringausfall an Fichtenbeständen. Wiss $Z$ Tech Univ Dresden 36, 273-275 